\title{
Hipotiroidismo congénito Evaluación neurológica y sicométrica
}

\author{
Dra. Andrea Gleisner A. ${ }^{1}$; Dr. Carlos Torres B. ${ }^{2}$; Dra, Vera Wilhelm P. ${ }^{1}$; \\ Dra. Sylvia Asenjo M.1 ; Sra. Andrea Adriazola M. ${ }^{3}$; Sra. Iris Cafati K. ${ }^{3}$; \\ Dr. Eduardo Valenzuela B. 4 ; Dr. Ricardo Lillo $G^{5}$
}

\author{
Congenital hypothyroidism \\ Neurologic and psichometric evaluation
}

\begin{abstract}
Twenty two children with congenital hypothyroidism (CHT) were studied in order to evaluate the occurrence of neurologic damage. Their disease was recognized on clinical grounds and subsequently confirmed by laboratory tests and diagnosis was dones usually late, most frecuently after several years, thus $95 \%$ of our cases had neurologic disturbances of variable severity, $91 \%$ had sub-normal IQ and $59 \%$ required special education.

(Key words: Hy pothyrojdism, congenital, age at diagnosis, mental subnormality).
\end{abstract}

La incidencia del hipotiroidismo congénito (HTC) es de 1 por 3.000 a 1 por 6.000 recién nacidos (RN) vivos ${ }^{1,2,3,4}$. La etiología mas frecuente corresponde a trastornos en el desarrollo de la glándula tirojdes, siendo el defecto en la hormonogénesis mucho más raro. El hipotiroidismo de origen hipotálamo-hipofisiario, aquel por resistencia periférica a hormonas tiroídeas, por ingestión de drogas antitiroideas durante el embarazo y el cretinismo endémico, son excepcionales.

Las manifestaciones clinicas en el RN son variables, el cuadro puede presentarse como un síndrome de hipotiroidismo franco con o sin bocio, o ser totalmente asintomático. En estos casos el diagnóstico es formulado por determinaciones hormonales sistemáticas.

Las secuelas neurológicas y el retardo mental constituyen el problema más relevante del HTC no tratado o tratado tardíamente ${ }^{5,6,6,8}$. En el presente estudio se evalúan estas secuelas en pacientes hipotiroideos, que al momento del diagnóstico presentaron manifestaciones clínicas de hipotiroidismo.

1. Endocrinología infantil, departamento ue pediatría, Universidada de Concepción.

2. Pediatra, servicio de pediattía, Hospital Guillermo Grant Benavente, Concepción.

3. Psicóloga. Centro de Diagnóstico, Ministerio de Eđucación. Concepción.

4. Servicio neurologia infantil, Hospital Guillermo Grant Benavente. Concepción.

5. Servicio medicina nuclear, Hospital Guillermo Grant Benavente. Concepción.

\section{MATERIAL Y METODO}

Se escudian las observaciones clínicas de 120 niños que se encuentran en control y tratamiento por hipotiroidismo congénito en el Policlínico de Endocrinología Infantil del Hospital Gmo. Grant Benavente de Concepçión.

Se incluyen en el trabajo los niños que cumplen los siguientes requisitos: edad mayor de 7 años, la que permite aplicar pruebas sicométricas; seguimiento regular en endocrinología infantil y cumplimiento de las indicaciones terapéuticas, El tiempo de control fluctúa entre 8 y 16 años, con un promedio de 10 años. Los datos del desarrollo sicomotor se obtienen del registro de la observación clínica; certificación del diagnóstico mediante determinación de concentraciones séricas de tirotoxina (T4) y tiroestimulina hipofisiaria (TSH). captación de I 131 y cintigrama tiroídeo.

La evaluación psicológica se efectúa en el Centro de Diagnóstico del Ministerio de Educación, por dos psicólogas clínicas quienes aplican la prueba de Wechsler Infantil Revisada (WIS-R), obteniendo los valores correspondientes de coeficiente intelectual (CI). La evaIuación neurológica se realiza por neurólogo infantil. Las determinaciones hormonales se hacen por radioinmunoanálisis (RIA). Se incluyen en el trabajo 22 niños que cumplen con los requisitos de selección.

\section{RESULTADOS}

Los pacientes seleccionados corresponden a $18 \%$ del total de niftos en control por HTC. Dieciocho nifios $(81,8 \%)$ son de sexo femenino.

El diagnóstico es planteado en sólo 2 niños (9\%) antes de los 3 meses y sólo en $6(27 \%)$ en el primer semestre de la vida. El diagnóstico más precoz se formula a los 40 días de vida y el más tardío a los 11 años de edad (Fig. 1).

Diecinueve niños son portadores de tiroides 


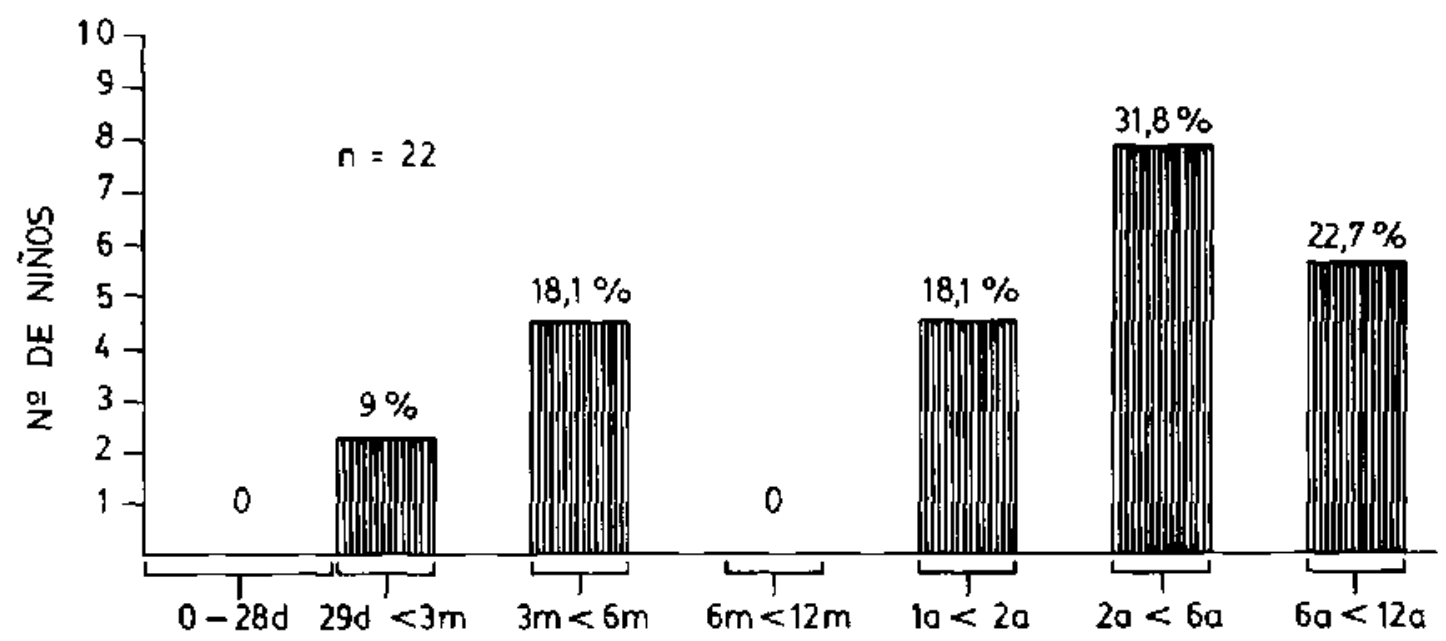

Figura 1. Hipotiroidismo congénito. Edad en el momento del diagnóstico.

ectópicos o disgeriéticos $(86,5 \%), 2$ presentan atireosis $(9 \%)$ y uno es portador de defecto de la hormonogénesis $(4.5 \%)$.

E] desarrollo sicomotor se encuentra en gene. ral retrasado y este retraso está en relación a la edad de diagnóstico (Tabla 1), La marcha se inicia alrededor de los 2 años en los niños diagnosticados antes de los 6 meses de vida $y$ a los 2 años 6 meses en aquellos diagnosticados después de los 2 años. En dos casos la marcha comienza recién a los 6 años. El lenguaje se inicia alrededor de $\operatorname{los} 2$ años en los niños diagnosticados antes de los 6 meses y en aquellos diagnosticados después de los 2 aftos de vida, comienza en promedio a los 3 años 6 meses. Tres niñós comienzan su lenguaje después de los 6 años de vida. Las alteraciones neurológicas están presentes en casi todos los niños, con excepción de 1 caso que tiene además un Cl normal. Los trastornos neurológicos más frecuentemente obser. vados son: alteración de los reflejos, torpeza motora gruesa y fina, trastornos del lenguaje, sincinesias, hipotonia, incoordinación y trastornos de conducta (Fig. 2).
El CI es normal en sólo 2 niños ( $9 \%$ ), normal lento en $5(23 \%)$ y limitrofe en $5(23 \%)$. Diez casos. (45\%) tienen un retardo mental evidente (Fig. 3)

Al correlacionar el CI con la escolaridad y su rendimiento escolar, es evidente que a medida que desciende el Cl total disminuye también el rendimiento escolar: Sólo en 9 niños (40\%) se obserya un rendimiento escolar satisfactorio y un niño tiene mal rendimiento en la educación básica regular; tres niños (13\%) asisten a grupo diferencial, seis nif̂os (26\%) están en escuela especial y tres njños no concurren a ningún tipo de escuela debido a su bajo CI y sus problemas de conducta (Tabla 2).

Cabe destacar que en los 2 cașos de atireosis el diagnóstico es formulado a $\operatorname{los} 3$ meses de vida, ambos inician la marcha a los 3 años de edad, uno de ellos presenta retardo mental leve y el otro, retardo mental medio.

\section{DISCUSION}

Las manifestaciones clínicas de hipoticoidismo

Tabla 1.

Inicio de la marcha y el tenguaje en relación a edad de diagnóstico

\begin{tabular}{|c|c|c|c|c|c|c|c|}
\hline \multicolumn{3}{|c|}{ Extad al diagnóstico } & $\begin{array}{l}N^{0} \cdot d e \\
\text { niños }\end{array}$ & $\begin{array}{l}\text { Edad } \\
\text { inicio }\end{array}$ & $\begin{array}{l}\text { Promedio } \\
\text { de marcha }\end{array}$ & $\begin{array}{l}\text { Edad } \\
\text { inicio }\end{array}$ & $\begin{array}{l}\text { Promedio } \\
\text { lenguaje }\end{array}$ \\
\hline $\mathbf{R N}$ & & -28 días & 0 & & 0 & & 0 \\
\hline 29 & días & - 3 meses & 2 & 1 año & 11 meses & 2 años & \\
\hline 3 & meses & -6 meses & 4 & 2 años & & 2 айоs & 3 meses \\
\hline 6 & meses & -12 meses & 0 & & 0 & & 0 \\
\hline 1 & año & - 2 años & 4 & 1 an̉o & 6 meses & 2 aก̃os & \\
\hline 2 & años & - 6 años & 7 & 2 aก̉os & 6 meses & 3 años & 6 meses \\
\hline 6 & años & -12 ànos & 5 & 2 años & 6 meses & 3 aกีos & 6 meses \\
\hline
\end{tabular}


REFLEJOS ALTERADOS

TORPEZA MOTORA FINA

TORPEZA MOTORA GRUESA

TRANST, LENGUAJE

SINCINESIAS

HIPOTONIA

INCOORDINACION

TRANST. CONOUCTA

TRANST. AUDICION

ALT. MARCHA

ESTRABISMO

NISTAGMUS

ALT. LATERALIDAD

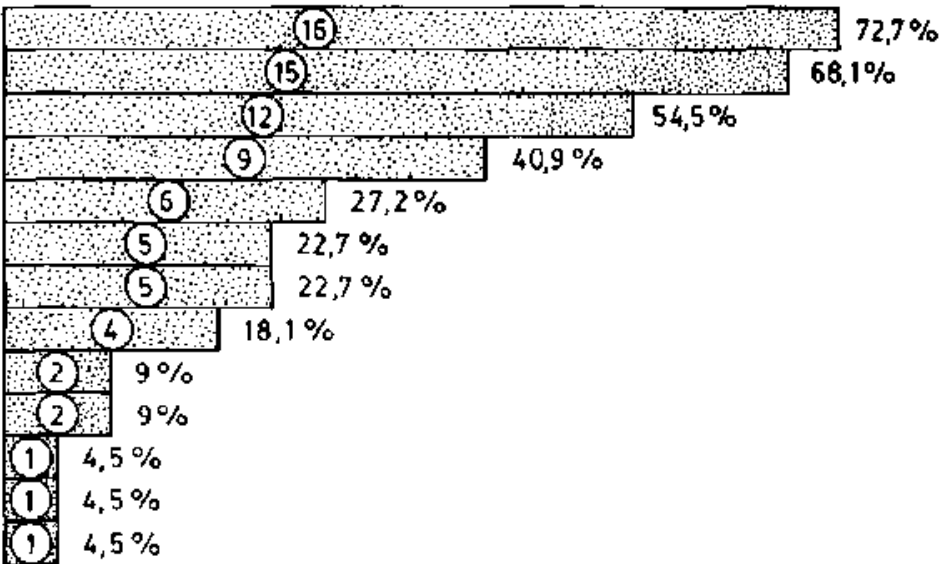

Figura 2. Hipotiroidismo congénito. Frecuencja de haljazgos neurológicos.

se presentan en forma gradual durante la infancia y aparecen más temprano en pacjentes con agenesia tiroidea que en aquellos que tienen un tiroides ectópico ${ }^{3,8}$. El diagnóstico formulado sobre una base clinica es por lo general más tardío y determina por lo tanto un pronóstico menos favorable, pudiendo estar relacionado con atireosis o con una insuficiencia tirojdea de larga evolución $6,7,8$.
La edad de diagnóstico basado en la sospecha clínica es variable según diferentes series: en Suecia, en el $20 \%$ de los casos de HTC se formula el diagnóstico al mes de vida ${ }^{8}$, en Dinamarca en el 10\% de ellos ${ }^{3}$ y en Estados Unidos en sólo el $2,9 \%$ de los $\operatorname{casos}^{9}$. Ninguno de nuestros niños es detectado antes de los 30 días de vida.

En las alteraciones neurológicas encontradas predominaron la torpeza motora gruesa y fina,

Tabla 2.

Relación entre coeficiente intelectual, escolaridad y tendimiento escolar

\begin{tabular}{|c|c|c|c|c|c|c|}
\hline \multirow[b]{2}{*}{ Paciente } & \multirow[b]{2}{*}{ Edad } & \multicolumn{2}{|c|}{ Coeficiente } & \multirow{2}{*}{$\frac{\text { Intelectual }}{\text { Total }}$} & \multirow[b]{2}{*}{ Escolaridad } & \multirow[b]{2}{*}{ Rendimiento } \\
\hline & & Verbal & Manual & & & \\
\hline C.P.Q. & $8 \mathrm{a} 11 \mathrm{~m}$ & 106 & 116 & 109 Normal & $4^{\circ}$ Básico & Bueno \\
\hline E.S.C. & $9 a$ & 97 & 87 & 92 Normal & $4^{\circ}$ Básico & Bueno \\
\hline R.S.C. & $8 a 9 m$ & 85 & 90 & 86 Normal lento & $3^{\circ}$ Básico & Aceptable \\
\hline I.A.T. * & $7 \mathrm{a} 9 \mathrm{~m}$ & - & - & - Normal lento & $1^{\circ}$ Básico & Regular \\
\hline C.M.Y.* & $8 \mathrm{a} \quad 8 \mathrm{~m}$ & - & - & - Normal lento & 10 Básico & Regular \\
\hline L.S.D. & $7 a \quad 8 m$ & 85 & 91 & 87 Nomal lento & $2^{\circ}$ Básico & Aceptable \\
\hline I.R.A. & $86 \mathrm{~m}$ & 91 & 82 & 85 Normal lento & $\begin{array}{l}2^{\circ} \text { Básico } \\
G \text {. Diferencial }\end{array}$ & Regular \\
\hline A.M.B. & $7 \mathrm{a} 5 \mathrm{~m}$ & 77 & 75 & 73 Limitrofe & $2^{\circ}$ Básico & Regular \\
\hline P.D.Q. & $7 a \quad 1 m$ & 84 & 72 & 76 Limítrofe & $\begin{array}{l}1^{\circ} \text { Básico } \\
\text { G. Diferencial }\end{array}$ & Regular \\
\hline C.G.D. & $7 \mathrm{a} 10 \mathrm{~m}$ & 82 & 78 & 79 Limítrofe & $\begin{array}{l}1^{0} \text { Básico } \\
\text { G. Diferencial }\end{array}$ & Regular \\
\hline B.B.Z. & $8 a 2 m$ & 81 & 75 & 75 Limítrofe & $3^{\circ}$ Básico & Satisfactorio \\
\hline B.S.B. & $12 \mathrm{a} 2 \mathrm{~m}$ & 79 & 73 & 75 Limitrofe & 50 Básico & Regulas \\
\hline V.S.P. & $9 \mathrm{a} 5 \mathrm{~m}$ & 69 & 65 & 65 R.M. leve & E. Especial & Satisfactorio \\
\hline A.V.G.* & $9 a$ & - & - & - R.M. medio & Ninguna & - \\
\hline G.S.S. & $12 a$ & 50 & 50 & 50 R.M. medio & E. Especial & Regular \\
\hline N.M.R. & $12 a$ & 50 & 50 & 50 R.M. medio & Ninguna & - \\
\hline M.C.C. & $9 \mathrm{a} 7 \mathrm{~m}$ & 57 & 51 & 52 R.M. medio & E. Especial & Malo \\
\hline O.B.E. & $11 \mathrm{a} 3 \mathrm{~m}$ & 55 & 67 & 58 R.M. medio & E. Especial & Malo \\
\hline M.A.M. & 9a $3 \mathrm{~m}$ & 63 & 58 & 57 R.M. medio & $1^{\circ} \mathrm{Básico}$ & Malo \\
\hline W.M.J. & $10 \mathrm{a}$ & 50 & 58 & 50 R.M. medio & E. Especial & Malo \\
\hline P.V.J.* & $11 a 9 m$ & - & - & - R.M. severo & Ninguna & - \\
\hline V.B.L. ${ }^{*}$ & $10 \mathrm{a} 10 \mathrm{a}$ & - & - & - R.M. severo & E. Especial & Malo \\
\hline
\end{tabular}

* Imposible evaluar por falta de cooperación del niño. 

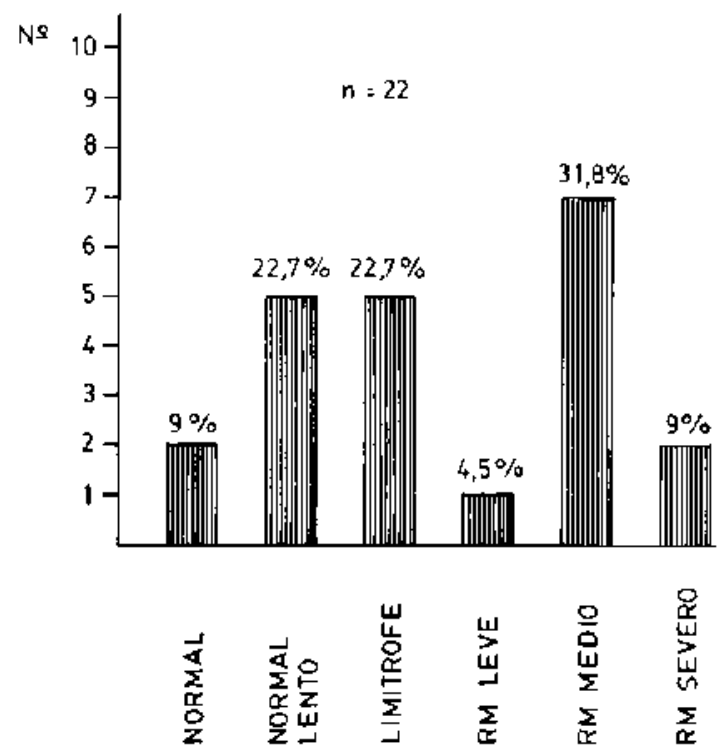

Figura 3. Hipotiroidismo congénito. Distribuciön según coeficiente intelectual.

trastornos de lenguaje y conducta, incoordinación y alteración de los reflejos profundos. Lesiones sugerentes de lesión cer ebral mínima pueden estar presentes aún en niños con un coeficiente intelectual normal $5,10,11$.

Jacobsen y Brandt en Dinamarca encuentran que $46 \%$ de niños hipotiroldeos diagnosticados por presentar signos y síntomas de insuficiencia tiroídea tienen un $\mathrm{Cl}$ inferior a $85^{3}$; Alm y cols. ${ }^{8}$ en Suecia encuentran un Cl promedio de 88 y que un $41 \%$ de los niños tienen CI menor de 85. El Cl promedio de los niños del presente estudio es de 71 , un $64 \%$ de ellos tienen un $\mathrm{CI}$ inferior a 85 y un $91 \%$ un CI menor de 90 . El $59 \%$ de los niños estudiados necesita educación especial, cifra muy superior al $23 \%$ descrito por los autores suecos ${ }^{8}$.

En el presente estudio la incidencia de alteraciones neurológicas y de retardo mental guardan correlación con la edad de diagnóstico y con la etiología de HTC. En los 2 casos de atireosis observamos retardo mental pese a que el diagnóstico fue formulado relativamente temprano, a los 3 meses de vida. Los factores más importantes en el pronóstico psicomotor de estos niños son la duración y severidad del hipotiroidismo fetal ${ }^{7}$. Los parámetros útiles para establecer la edad (fetal) del inicio del cuadro serían la edad ósea ${ }^{6,10}$ y la determinación de alfa--fetopro. teínas según ha sugerido recientemente Larsson y cols. ${ }^{12}$. La presencia de signos y sintomas al nacer refleja la severidad del hipotiroidismo y es probable que estos niños tengan mal pronóstico a pesar del tratamiento sustitutivo precoz ${ }^{6,8}$.

La pesquisa sistematica del HTC en el $\mathrm{RN}$ ha permitido el diagnóstico $y$ tratamiento precoz de esta afección. Los coeficientes intelectuales normales en pacientes tratados en forma adecuada $y$ antes de presentar manifestaciones clínicas, confirman la importancia del tratamiento precoz de la insuficiencia tiroídea ${ }^{6,7}$.

\section{RESUMEN}

Se analizan 22 nifios portadores de HTC, cuyo diagnóstico se formula sobre bases clínicas y se confirma posteriormente por exámenes de laboratorio. El $95 \%$ de los niños presenta alteraciones neurologicas de intensidad variable, el $91 \%$ tiene un $\mathrm{CI}$ inferior al normal y el $59 \%$ de ellos necesita educación especial.

\section{REFERENCIAS}

1. Price, $D$, Ehrich, $R$. and Walfish, P.: Congenital hypothyroidism. Clinical and laboratory characteristics in infants detected by neonatal screening. Arch, Dis. Child, 56: 845, $198 \mathrm{I}$.

2. Rochiccioli, $P$, Dutar, G.: Le depestage neonatal de l'hypothyroidie, Arch. Franc. Pédiat. 36: 7, 1979.

3. Jacobsen, B., Brandt, N.: Congenital hypothyroidism in Dennmark. Arch. Dis. Child. 56: 134, 1981.

4. Larsson, A., Ljunggren, J.G., Ekman, $K_{1}$ Nilsson, A., Olin, P.: Scteening for congenital bypothyroidism. Acta Paediatr. Scand. 70: 141, 1981.

5. Virtonen, M., Maenpaa, J., Santaviori, P, Hirzonen, $E$. Perhecntupa, $J .:$ Congenital Hypothyrojdism: Age at start of treatment versus outcome. Acta Paediatr. Scand. 72: 197, 1983.

6. New England Congenital Hyporhyroidism Collaborative. Effects of neonatal screening for hypothy. roidism: Prevention of mental retardation by treatment before clinical manifestations. Lancet 2: 1095, 1981

7. Glorieux, J., Dussoulf, J, Letarte, J., Guyda, H., Morissette, $F_{\text {.. }}$ Preliminary results on the mertal development of hypothyroid infants detected by the Quebec Screening Program, J. Pediatr. 102: 19, 1983.

8. Alm, J., Larsson, A., Zetterström, R. Congenital Hypothyroidism in Sweden. Acta Paediatr. Scand. 70: $907,1981$.

9. Fisher, D.A., Dussault. J.H., Foley T.P. et al: Screening for congenital hypothyroidism: Results of screening one million north american infants, $J$, Pediatr, 94: 700, 1979.

10. Walter, R., Noel, P., De Cock, P, et al: Neuropsychological study in treated thyroid dysgenesis. Acta Paediatr. Scand. (Suppl). 277: 41, 1980.

11. Mac Faul, R., Dornes, S., Brett, E., Grant, D.: Neurological abnormalities in patients treated for hypothyroidism from arly life. Arch. Dis. Child. 53: 611, 1978 .

12. Larson, A., Hagenfeldt, L., Blom. L., Mortensson, W.: Serum a-Fetoprotein - A biochemical indicator of prenatal hypothyroidism. Acta Paediatr. Scand. $72: 481,1983$. 